\title{
Psychological Aspects of COVID-19 in Iran: How the Disease May Affect Mental Health of Medical Staff and General Population?
}

\author{
Mohsen Saffari ${ }^{1,2}{ }^{\circledR}$, Mehdi Raei $^{1,3}{ }^{\circledR}$, Mohamad Amin Pourhoseingholi $^{\circledR}$, Masoum Khosh Fetrat ${ }^{5^{*}}$ \\ ${ }^{1}$ Health Research Center, Life Style Institute, Baqiyatallah University of Medical Sciences Tehran, Iran \\ ${ }^{2}$ Health Education Department, Faculty of Health, Baqiyatallah University of Medical Sciences, Tehran, Iran \\ ${ }^{3}$ Department of Epidemiology and Biostatistics, Faculty of Health, Baqiyatallah University of Medical Sciences, Tehran, Iran \\ ${ }^{4}$ Gastroenterology and Liver Diseases Research Center, Research Institute for Gastroenterology and Liver Diseases, Shahid Beheshti \\ University of Medical Sciences, Tehran, Iran \\ ${ }^{5}$ Department of Anesthesiology and Critical Care, Khatamolanbia Hospital, Zahedan University of Medical Sciences, Zahedan, Iran
}

Corresponding Author: Masoum Khosh Fetrat, MD, Associate Professor, Department of Anesthesiology and Critical Care, Khatamolanbia Hospital, Zahedan University of Medical Sciences, Zahedan, Iran.Tel: +98-9196017138, EMail: drkhoshfetrat@yahoo.com

Received December 13, 2020; Accepted January 4, 2021; Online Published January 20, 2021

\begin{abstract}
Introduction: The disease related to the coronavirus disease 2019 (COVID-19) has now been a pandemic throughout the world. Although the epidemiological studies and clinical trials are utilized to find standard measures and medicines to prevent and control COVID-19, addressing the mental health and psychology of the people who may be at risk of the disease may also be effective to find comprehensive methods to better overcome this pandemic. This study aimed at investigating the mental status of both the general population and healthcare personnel during this pandemic in Iran.

Methods: Using a cross-sectional design, a sample of 296 medical staff and 532 people living in Tehran city of Iran were included in the study. The assessment was performed by Depression, Anxiety, and Stress scale short-form (DASS-21). Two groups were matched in terms of some demographical factors such as age, gender, and marital status. Analysis of covariance (ANCOVA) was utilized to perform comparisons between the two groups.

Results: Both samples of the general population and medical staff had high levels of stress, anxiety, and depression. The mean score of anxiety and depression among medical staff was higher than that of the general population $(P<0.001)$. There was no significant difference between the two groups in terms of stress level.

Conclusion: It seems both the Iranian general population and healthcare personnel are under mental pressure caused by COVID-19, indicating an urgent need for intervention and using preventive measures to control the negative impacts of the diseases on the psychological well-being of these people. Further studies in other regions affected by the disease may provide more evidence in this regard.

Keywords: Coronavirus, COVID-19, Mental Health, Depression, Anxiety, Stress
\end{abstract}

Citation: Saffari M, Raei M, Pourhoseingholi MA, Khosh Fetrat M. Psychological aspects of COVID-19 in Iran: how the disease may affect mental health of medical staff and general population? Int J Travel Med Glob Health. 2021;9(2):94-99. doi:10.34172/ijtmgh.2021.15.

\section{Introduction}

New coronavirus disease (COVID-19) has emerged firstly from Wuhan, China in December 2019. ${ }^{1}$ The disease quickly spread around the world and by April 2020 approximately all of the countries reported some new cases. The virus seems to be controlled in China but it has now been changed to a pandemic according to World Health Organization (WHO). ${ }^{2}$ Unfortunately to date (April 2020) more than 1400000 people (with nearly 81000 deaths) at global level have been infected by the COVID-19, and many European countries, as well as the United States, have been reported as new regions with the highest occurrence of the disease. ${ }^{1,3,4}$ Among some Asian countries such as China, Japan, and South Korea, the measures to control and prevent the disease have led to promising results; however, some other countries, including Iran has no specified trend of the disease, and it has been estimated due to some social and economic problems (i.e., some cultural aspects that may negatively affect preventive

Copyright (C) 2021 The Author(s). This is an open-access article distributed under the terms of the Creative Commons Attribution License (http:// creativecommons.org/licenses/by/4.0), which permits unrestricted use, distribution, and reproduction in any medium, provided the original work is properly cited. 
recommended measures and International sanctions imposed mostly by the United States) the management of the disease and it's controlling may not happen in a near future. ${ }^{2,5}$ Also, shortages of medical staff especially nursing personnel as well as inadequate financial resources to supply medicines and medical equipment to provide standard healthcare for patients have worsened and complicated the prediction of the disease. Thus, these conditions along with the unknown nature of the disease and unavailability of an approved vaccine or medicine to prevent and control COVID-19 changed it to a phobia in the Iranian population. ${ }^{6}$

The fear of a fatal disease is not surprising and reports from other epidemic diseases especially those which may be transmitted through respiratory infections such as the Middle East respiratory syndrome (MERS) and severe acute respiratory syndrome (SARS) that both developed by other types of coronaviruses indicate when an outbreak/epidemic could not be controlled and there is no vaccine or medicine to protect the community, it generally affects the mental health of the population and may lead to the prevalence of mental disorders such as phobia, panic attacks, post-traumatic stress disorder, obsessive-compulsive conditions, suicide, etc..$^{7-9}$ However, the situation regarding the COVID-19 is somehow different from previous epidemics of coronaviruses because this has now been converted to a pandemic and its virulence and mortality have been evaluated multiple times higher than previous outbreaks or epidemics that has happened before. ${ }^{10,11}$ The situation not only affects the general population as a mental concern but also the medical staff as the front lines to fight the disease may be influenced even more seriously.

To date, unfortunately, there are a few studies that have addressed the mental health and psychological aspects of the disease, whereas these conditions may develop a long term effect of the disease across the community and act as inhibitors against medical measures targeted improvements of physical conditions of the patients and healthy people. Actually, when the disease changes the attitude and hope in people to overcome the disease and due to high levels of stress and anxiety they may not adjust themselves to the preventive measures and recommendations, this may have a negative and irreversible impact on social coherence and even medical staff commitment to control the disease and finally may contribute to more frequency of the disease through the community. ${ }^{12,13}$ Therefore, assessing the mental health situation of the community as well as medical staff regarding the disease may help to clarify the current situation and show necessary measures needed to prevent and control these side effects of the disease, as well.

A suggested way to control the transmission of the disease in the community is home quarantine. The situation also may impose new stressors and mental pressures on people who are in quarantine that may increase the general psychological difficulties for the community. ${ }^{14}$ By the situation, the people should change their activities of daily livings and they must isolate themselves in their homes. Hence similar to people who are in a self-made prison, the people must deprive themselves of many enjoyable activities and entertainments that are necessary for a sense of well-being. ${ }^{15}$ Moreover, medical staff that are responsible to control and manage the disease may also have a similar experience. They should spend most of their time in the clinics and hospitals and they also may be deprived of their routine activities, including the time that they may be along with their families.

Nevertheless, besides the epidemiological studies and clinical trials to find standard measures and medicines to prevent and control COVID-19, addressing the mental health and psychology of the people who may be at risk of the disease may also be effective to find comprehensive methods to better overcome this struggle and provide hope and willingness for people in the community and medical staff to eradicate this virus. Therefore, we decided to assess how the disease may affect the psychological condition of the general population as well as medical staff in a preliminary study.

\section{Methods}

\section{Study Design and Setting}

This was a cross-sectional study conducted, in FebruaryMarch 2020 from a sample of medical staff who worked in Baqiyatallah Hospital, Tehran, Iran. All participants were informed about the aims of the study prior to data collection, and their written (medical staff) or verbal (general population) informed consent was obtained.

\section{Study Population}

Baqiyatallah Hospital is one of the main and referral centers for specialized diagnosis and treatment of patients with COVID-19 in Tehran. Therefore, many participants were selected from this center and a number of working medical staff from two other medical centers that had admitted these patients were included, as well. Also, a sample of general population who lived in the central area of Tehran, Iran (where the hospitals were located) was included in the study. The general population was matched based on three factors of sex (similar percent of male and female), age (with an average difference of one year), and marital status (similar percent of married and unmarried). Thus, only those who had similar conditions in terms of such variables have been included. Eligibility of the general population also was identified as living in the vicinity of the selected hospitals and agreement to participate by phone contact. To select a sample of general population, a phone list of people who had medical records in the hospitals was received and a call interview was planned to collect information. An expert nurse who was oriented to phone screening of the COVID-19 contacted the families and after the screening of symptoms of the disease on the phone call, asked participants to answer some questions regarding their mental health. If the participant agreed, the questionnaire was completed by one or two members of that family.

\section{Sample Size and Sampling Methods}

To calculate sample size for both groups, the formula suggested by Hulley et $\mathrm{al}^{16}$ was used to indicate the sample size by an odds ratio of an outcome (i.e., anxiety). Hence, with the 
parameters: $\alpha=0.05, \beta=0.2$, q1 (Proportion of subjects that are in group 1$)=(0.5)$ and odds ratio (in group 2$)=1.5$, the sample sizes for medical staff and general population groups were 296 and 532, respectively. Sampling was convenient, so that all people who were eligible and were accessible could participate in the study.

\section{Inclusion and Exclusion Criteria}

Inclusion criteria for medical staff were healthcare responsibility for patients with the absolute diagnosis of COVID-19 or suspected patients whose diagnosis has not been confirmed but have been under investigation for at least two weeks, and have accepted to participate in the study. Those medical staff who might be affected by the disease based on clinical manifestation or laboratory tests were excluded. Individuals who had experiences of disease symptoms were invited to a nearby hospital for further investigation; however, they were excluded from the study. In addition, those without any history of psychological distresses such as panic attacks, posttraumatic stress disorder (PTSD), major depression, and other severe mental health disorders (based on self-report on a phone call) were included in the study.

\section{Data Measurement Tools}

To identify the psychological factors, we used the Depression, Anxiety, Stress Scale questionnaire (DASS-21), which is a short form of a 42 -item scale that assesses the symptoms of depression, anxiety, and stress in people over the past week (each one assessed by seven items). The depression subscale includes items that determined dysphoria, hopelessness, anhedonia, devaluation of life, and inertia; The anxiety subscale evaluated acute responses to fear and subjective symptoms of anxiety, and the stress subscale contains items that measure tension, irritability, and difficulty in relaxing. ${ }^{17}$ The scale asks participants to answer each item based on options from "did not apply to me" (0) to "applied to me very much" (3) and to compute the total score for each section (i.e., depression, anxiety, and stress) the summed of all individual scores related to that section should be multiplied by two. Thus, the total score for each item may range from 0 to $42 .{ }^{18}$ Although the scale has not been designed for diagnostic purposes, it may be useful to assess mental disturbances in an adult population. Based on suggested cut-points, any section could be categorized into five categories from normal to extremely severe in term of severity. The Persian version of the scale with appropriate psychometric properties was used in our study. ${ }^{19}$

\section{Data Collection}

DASS-21 questionnaire and the demographic data sheet were filled out by all participants. Demographic variables included age, gender, and, marital status. Furthermore, work experience (years) and the experience working with COVID-19 patients (weeks) were recorded for medical staff. The study participants were all informed about the objectives of the study and written informed consent was obtained from each participant. They were also assured of confidentiality.

\section{Data Analysis}

A multivariate analysis of variance was performed to investigate group differences (community population vs medical staff) in psychological well-being indices. Three dependent variables were used, namely anxiety, stress, and depression. The independent variable was the group to which participants belonged and the covariates were age, gender, and marital status. Preliminary testing was conducted to check for the model's assumptions; the Box's test of equality of covariance matrices was performed to test the multivariate normal distribution and equality of variance-covariance matrices. Mahalanobis distances were calculated to test for outliers. In addition, the correlation coefficients between the dependent variables were calculated in order to test the multicollinearity assumption. All statistical analyses were performed using SPSS version 22 and all $P$ values $<0.05$ were considered statistically significant.

\section{Results}

A total of 296 medical staff and 532 people from the general population took part in this study. The mean age \pm standard deviation (SD) of participants was 38.78 \pm 6.95 (39.28 \pm 8.08 for the general population vs $38.50 \pm 6.20$ for medical staff). Most participants were male $(60.2 \%$ for the general population vs. $59.9 \%$ for medical staff), and most of them were married $(62.7 \%$ for the general population vs. $65.6 \%$ for medical staff). There were distinguishable differences among the participant groups involved in the study regarding anxiety and depression. The results showed that there was no significant difference between males and females $(P=0.947)$, married and unmarried $(P=0.45)$, and age $(P=0.16)$ between people on the combined dependent variables (Table 1 ).

The maximum value for Mahalanobis distance (14.01) was less than the critical value (16.27), hence there were no substantial multivariate outliers and checking other assumptions proceeded. Box's test of equality of covariance matrices demonstrated that the data had multivariate normal distribution and homogeneity of variance. The dependent variables were only moderately correlated; there was no multicollinearity between the variables (Table 2).

There was a statistically significant difference between community population and medical staff groups on the combined dependent variables, $\mathrm{F}(6,1318)=142, P<0.001$; Pillai's trace $=0.78$; partial eta squared $=0.39$. When the results for the dependent variables were considered separately, the

Table 1. Demographic Characteristics, Results of Stress, Anxiety, and Depression Scores Based on study Groups

\begin{tabular}{lccc}
\hline Variables & $\begin{array}{c}\text { Community Population } \\
(\mathbf{N}=\mathbf{2 9 6})\end{array}$ & $\begin{array}{c}\text { Medical staff } \\
(\mathbf{N}=\mathbf{5 3 2})\end{array}$ & $\boldsymbol{P}$ Value \\
\hline Male (No. ,\%) & $145(60.2)$ & $254(59.9)$ & 0.947 \\
Married (No. ,\%) & $151(62.7)$ & $278(65.6)$ & 0.45 \\
\hline Variables & Mean \pm SD & Mean \pm SD & \\
\hline Age & $39.28 \pm 8.08$ & $38.50 \pm 6.20$ & 0.160 \\
Stress & $27.34 \pm 4.37$ & $27.58 \pm 5.29$ & 0.53 \\
Anxiety & $26.04 \pm 4.52$ & $27.33 \pm 4.61$ & $<0.001$ \\
Depression & $26.09 \pm 4.39$ & $27.73 \pm 5.03$ & $<0.001$ \\
\hline
\end{tabular}


Table 2. Pearson Correlation Values Between Dependent Variables

\begin{tabular}{lccc}
\hline & \multicolumn{3}{c}{ Pearson Correlations $(\boldsymbol{P}$ Value $)$} \\
\cline { 2 - 4 } & Stress & Anxiety & Depression \\
\hline Stress & 1 & $0.12(0.002)$ & $0.07(06)$ \\
Anxiety & & 1 & $0.13(0.001)$ \\
Depression & & & 1 \\
\hline
\end{tabular}

differences to reach statistical significance, using a Bonferroni adjusted alpha level of 0.017 , were stress $(P=0.53)$, anxiety $(P<0.001)$, and depression $(P<0.001)$. An inspection of the mean scores indicated that medical staff reported slightly higher levels of anxiety and depression than the community population, while the stress indicated no significant difference (Table 1 and Figure 1).

\section{Discussion}

The current study was conducted to examine the level of stress, anxiety, and depression in medical staff as well as community people while a fearful and disastrous virus (new coronavirus) has now been pandemic around the world, especially in Iran. We found there may be an abnormal mental health situation both between the general population and medical staff and medical staff may further suffer from symptoms of anxiety and depression compared to general population. In average, the scores indicate both groups are in severe categories of all stress, anxiety, and depression suggesting further assessment and immediate interventions prevent serious psychological disturbances, especially for healthcare personnel.

There are limited studies that have addressed mental health conditions among the general population and medical staff during the pandemic of COVID-19 especially in countries other than China. ${ }^{20-23} \mathrm{Li}$ et al attempted to assess the psychological consequences of the disease using users of a social network (Webio). They assessed posts of more than 17500 users based on machine-learning predictive models, and word frequency and emotional indicators of psychological concepts such as anxiety, depression, indignation, and happiness were examined before and after declaration of COVID-19 in January 2020. They found negative emotions, including stress, anxiety, and depression have been increased and positive states such as happiness and life satisfaction have been decreased considerably between these two time periods. Also, the serious concerns of people regarding the health of themselves and their family as well as a reduction in leisure time were notable in this study. ${ }^{13}$

In a study, Cao et al investigated the psychological impact of COVID-19 on students of Changzhi Medical College located in China. They used the generalized anxiety disorder scale and found that nearly one percent of respondents were experiencing severe anxiety and about $3 \%$ to $21 \%$ of participants had mild to moderate anxiety. ${ }^{24}$ In another study, immediate psychological responses of the disease in the initial stage of its epidemic were assessed in a general population in China. An online survey from 31 January to 2 February 2020 was performed and information on mental health was collected by Impact of Event Scale, and DASS-21

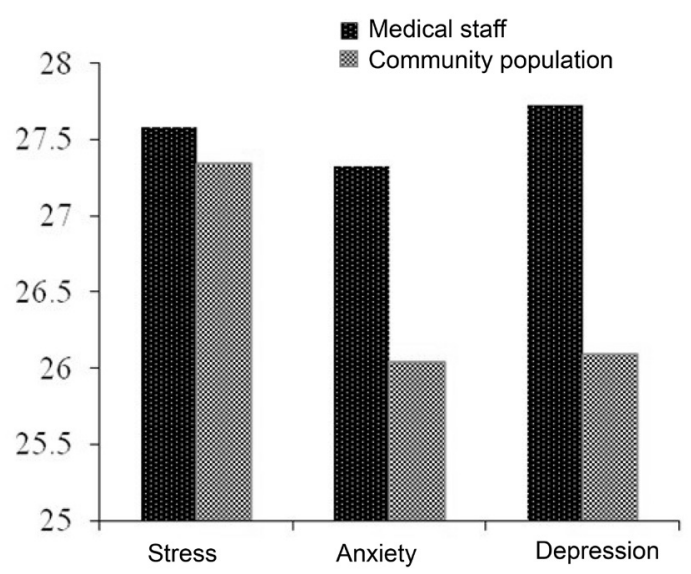

Figure 1. Comparison Between Scores of Stress, Anxiety, and Depression in Medical Staff and the General Population.

from 1210 respondents. More than half of the participants have appraised the psychological impact of the disease as moderate to severe and $16.5 \%$ to $28.8 \%$ reported moderate to severe depressive and anxiety symptoms. However, only $8 \%$ of subjects reported moderate to severe stress level. Also, more than $80 \%$ of them were worried about the likelihood of their family contacting COVID-19. ${ }^{25}$ Liu et al also reported the prevalence of posttraumatic stress symptoms and sleep quality in the hardest-hit area in China one month after the epidemic of the disease. They surveyed 285 residents and found that the prevalence of PTSD was 7\%, whereas the sleep quality of assessed people also have been influenced and many participants reported insomnia and sleeping problems because of concerns regarding the potential morbidity with COVID $-19 .{ }^{26}$

All studies presented here indicated consistency with our findings stating the disease has a strong impact on the mental health of both the general population and medical staff. However, since many of these studies are related to primary phases of COVID-19 epidemic in China in which the risks of the disease, its complications, and mortality have not been identified exactly, the levels of psychological disturbances may be evaluated somehow lower than that of our study that is not surprising. We believe new investigations in countries that are now mostly affected by the disease and high numbers of contacted people as well as high mortality rates will show the increasing negative impacts of the disease on emotions and psychological well-being of the population and healthcare workers, as well.

Comparing the results of the current study to those of other studies that reported psychological status among medical staff also confirmed that there are now significant increments of stress, anxiety, and depression among Iranian healthcare workers compared to the past that may be attributed to COVID-19. For example, Saeedi Shahri et al in a systematic review examined depression among Iranian nurses. They assessed 30 studies in this regard and found that the pooled prevalence of mild to moderate depressive symptoms among Iranian nurses was $22 \%$ and only $5 \%$ reported severe depression in this group. ${ }^{27}$ In another systematic review, Mata 
et al assessed depressive symptoms among resident physicians. They extracted results of 31 cross-sectional studies as well as 23 longitudinal ones and found that only $28.8 \%$ of studies had reported moderate to severe such symptoms among the physicians. ${ }^{28}$

The current study has some limitations that should be considered before any interpretation or generalization of findings. Firstly, we only included participants from one hospital located in Tehran and general population around this healthcare center; therefore, the findings may not be generalized to other cities or countries. However, we believe because there is imitated understanding regarding the COVID-19 and its effects on mental health of population and healthcare personnel, this preliminary study may be helpful in this regard. Secondly, we only matched three factors of gender, age, and marital status in our groups, whereas there are many other factors in both groups that may affect the mental health status and due to some barriers we could not measure them here. Thirdly, our samples were selected employing a convenience sampling method and many people who might be in bad conditions of psychological status could not participate in the study, and including them may change the current findings. Finally, only variables such as depression, anxiety, and stress as well as a few demographics have been evaluated, while considering more comprehensive variables related to both socioeconomic status and mental health such as happiness, self-efficacy, locus of control, selfesteem, and the like may provide a clearer picture of mental health conditions among the study populations and would be helpful to find associated factors with mental health.

\section{Conclusion}

Findings revealed that many general population and medical staff may be at risk of mental health disorders during the pandemic of COVID-19 in Iran. Therefore, planning to proper use of preventive measures and improving the psychological status of both community people and healthcare staff are needed in response to negative impacts of the disease among the general population and medical staff who are fighting against the disease. Further investigations in other regions of the world and countries which are in focal areas of this pandemic also are necessary to provide a more precise perspective of the negative impacts of the disease both in affected people and healthy ones. Designing some psychological interventions and education programs to relieve these negative outcomes also should be considered.

\section{Authors' Contributions}

MS, MKF, and MAP developed the study concept. All authors contributed to the study design. Testing and data collection were performed by MR, MKF, and MAP. Data analysis and interpretation were performed by MR and MAP. All authors approved the final version of the manuscript for submission.

\section{Conflict of Interest Disclosures}

The authors report no known conflict of interest.

\section{Research Highlights}

\section{What Is Already Known?}

The previous published related studies revealed high levels of anxiety, stress, and depression among Iranian populations during the pandemic of COVID-19.

\section{What Does This Study Add?}

The current study demonstrated high levels of stress, anxiety, and depression among the general population and medical staff; anxiety and depression levels were higher in medical staff compared to that of general population. The results revealed that more mental hygiene monitoring and assessment are required in the COVID-19 era.

\section{Ethical Approval}

This study was approved by the Baqiyatallah University of Medical Sciences Ethics Committee, Tehran, Iran, with the code of IR.BMSU. REC.1398.442. Informed consent was obtained from all participants.

\section{Funding/Support}

This research did not receive any specific grant from funding agencies in the public, commercial, or not-for-profit sectors.

\section{Acknowledgments}

The authors would like to thank the "Clinical Research Development Unit" of Baqiyatallah Hospital for helping with timely data collection. Meanwhile, all healthcare personnel who contributed to conducting this study are appreciated.

\section{References}

1. Park M, Cook AR, Lim JT, Sun Y, Dickens BL. A systematic review of COVID-19 epidemiology based on current evidence. J Clin Med. 2020;9(4):967. doi:10.3390/jcm9040967.

2. Coronavirus Disease 2019 (COVID-19): Situation Report - 72. World Health Organization website 2020. https://apps.who.int/ iris/handle/10665/331685. Published 2020. Accessed March 4, 2020.

3. Zhang X. Epidemiology of COVID-19. N Engl J Med. 2020; 382(19):1869. doi:10.1056/NEJMc2005157.

4. Lai CC, Wang CY, Wang YH, Hsueh SC, Ko WC, Hsueh PR. Global epidemiology of coronavirus disease 2019 (COVID-19): disease incidence, daily cumulative index, mortality, and their association with country healthcare resources and economic status. Int J Antimicrob Agents. 2020;55(4):105946. doi:10.1016/j.ijantimicag.2020.105946.

5. Takian A, Raoofi A, Kazempour-Ardebili S. COVID-19 battle during the toughest sanctions against Iran. Lancet. 2020;395(10229):1035-1036. doi:10.1016/s01406736(20)30668-1.

6. Ahorsu DK, Lin CY, Imani V, Saffari M, Griffiths MD, Pakpour AH. The fear of COVID-19 scale: development and initial validation. Int J Ment Health Addict. 2020:1-9. doi:10.1007/ s11469-020-00270-8.

7. Jeong H, Yim HW, Song YJ, et al. Mental health status of people isolated due to Middle East Respiratory Syndrome. Epidemiol Health. 2016;38:e2016048. doi:10.4178/epih.e2016048.

8. Lung FW, Lu YC, Chang YY, Shu BC. Mental symptoms in different health professionals during the SARS attack: a followup study. Psychiatr Q. 2009;80(2):107-116. doi:10.1007/s11126- 
009-9095-5.

9. Park JS, Lee EH, Park NR, Choi YH. Mental health of nurses working at a government-designated hospital during a MERSCoV outbreak: a cross-sectional study. Arch Psychiatr Nurs. 2018; 32(1):2-6. doi:10.1016/j.apnu.2017.09.006.

10. Adhikari SP, Meng S, Wu YJ, et al. Epidemiology, causes, clinical manifestation and diagnosis, prevention and control of coronavirus disease (COVID-19) during the early outbreak period: a scoping review. Infect Dis Poverty. 2020;9(1):29. doi:10.1186/s40249-020-00646-X.

11. Jin Y, Yang H, Ji W, et al. Virology, epidemiology, pathogenesis, and control of COVID-19. Viruses. 2020;12(4):372. doi:10.3390/ v12040372.

12. Duan $\mathrm{L}, \mathrm{Zhu}$ G. Psychological interventions for people affected by the COVID-19 epidemic. Lancet Psychiatry. 2020;7(4):300302. doi:10.1016/s2215-0366(20)30073-0.

13. Li S, Wang Y, Xue J, Zhao N, Zhu T. The impact of COVID-19 epidemic declaration on psychological consequences: a study on active weibo users. Int J Environ Res Public Health. 2020; 17(6):2032. doi:10.3390/ijerph17062032.

14. Liu JJ, Bao Y, Huang X, Shi J, Lu L. Mental health considerations for children quarantined because of COVID-19. Lancet Child Adolesc Health. 2020;4(5):347-349. doi:10.1016/s23524642(20)30096-1.

15. Jiménez-Pavón D, Carbonell-Baeza A, Lavie CJ. Physical exercise as therapy to fight against the mental and physical consequences of COVID-19 quarantine: special focus in older people. Prog Cardiovasc Dis. 2020;63(3):386-388. doi:10.1016/j. pcad.2020.03.009.

16. Hulley SB, Cummings SR, Browner WS, Grady DG, Newman TB. Designing Clinical Research: An Epidemiologic Approach. 4th ed. Philadelphia: Lippincott Williams \& Wilkins; 2013.

17. Lovibond SH, Lovibond PF. Manual for the Depression Anxiety Stress Scales. Sydney, NSW: Psychology Foundation of Australia; 1995.

18. Lovibond PF, Lovibond SH. The structure of negative emotional states: comparison of the Depression Anxiety Stress Scales (DASS) with the Beck Depression and Anxiety Inventories. Behav Res Ther. 1995;33(3):335-343. doi:10.1016/00057967(94)00075-u.
19. Asghari A, Saed F, Dibajnia P. Psychometric properties of the Depression Anxiety Stress Scales-21 (DASS-21) in a nonclinical Iranian sample. Int J psychol 2008;2(2):82-102.

20. Sani G, Janiri D, Di Nicola M, Janiri L, Ferretti S, Chieffo D. Mental health during and after the COVID-19 emergency in Italy. Psychiatry Clin Neurosci. 2020;74(6):372. doi:10.1111/ pcn.13004.

21. Fiorillo A, Gorwood P. The consequences of the COVID-19 pandemic on mental health and implications for clinical practice. Eur Psychiatry. 2020;63(1):e32. doi:10.1192/j. eurpsy.2020.35.

22. Greenberg N, Docherty M, Gnanapragasam S, Wessely S. Managing mental health challenges faced by healthcare workers during covid-19 pandemic. BMJ. 2020;368:m1211. doi:10.1136/ bmj.m1211.

23. Torales J, O’Higgins M, Castaldelli-Maia JM, Ventriglio A. The outbreak of COVID-19 coronavirus and its impact on global mental health. Int J Soc Psychiatry. 2020;66(4):317-320. doi:10.1177/0020764020915212.

24. Cao W, Fang Z, Hou G, et al. The psychological impact of the COVID-19 epidemic on college students in China. Psychiatry Res. 2020;287:112934. doi:10.1016/j.psychres.2020.112934.

25. Wang $\mathrm{C}$, Pan $\mathrm{R}$, Wan $\mathrm{X}$, et al. Immediate psychological responses and associated factors during the initial stage of the 2019 coronavirus disease (COVID-19) epidemic among the general population in China. Int J Environ Res Public Health. 2020;17(5). doi:10.3390/ijerph17051729.

26. Liu N, Zhang F, Wei C, et al. Prevalence and predictors of PTSS during COVID-19 outbreak in China hardest-hit areas: gender differences matter. Psychiatry Res. 2020;287:112921. doi:10.1016/j.psychres.2020.112921.

27. Saeedi Shahri SS, Ghashghaee A, Behzadifar M, et al. Depression among Iranian nurses: a systematic review and metaanalysis. Med J Islam Repub Iran. 2017;31:130. doi:10.14196/ miri.31.130.

28. Mata DA, Ramos MA, Bansal N, et al. Prevalence of depression and depressive symptoms among resident physicians: a systematic review and meta-analysis. JAMA. 2015;314(22):23732383. doi:10.1001/jama.2015.15845. 